\title{
Low-Cost and Ultra-Low-Power Consuming RTUs for Use in IoT Systems
}

\author{
Ivan Ganchev, Zhanlin Ji, and Máirtín O’Droma
}

\begin{abstract}
This paper presents the design and realization of lowcost and ultra-low-power consuming remote transfer units (RTUs), working as communication gateways for collecting, aggregating, and forwarding IoT data to information centers (servers) in the cloud for further processing and data mining. Two types of RTUs, targeting different application scenarios and utilizing different communication standards, were designed - one, based on the General Packet Radio Service (GPRS) standard, and another - on the NarrowBand Internet of Things (NB-IoT) standard. The developed RTUs were experimentally tested and their use was successfully demonstrated in different IoT systems.
\end{abstract}

Keywords - hardware design, Internet of Things (IoT), Remote Transfer Unit (RTU), ultra-low power consumption.

\section{INTRODUCTION}

$\mathrm{T}_{\mathrm{i}}^{\mathrm{H}}$ HE Internet of Things (IoT) is seen as another information and industrial wave following those of personal computers, the Internet, and mobile communication networks [1]. It is becoming the main driving force of the future global economic development. The worldwide IoT market will soon reach $\$ 1.7$ trillion compared to $\$ 655.8$ billion in 2014, with a compound annual growth rate of $16.9 \%$ [1].

The Remote Transfer Units (RTUs), a.k.a. Data Transfer Units (DTUs), are the key modules in the IoT systems. For wireless communication, RTUs may utilize different standards and different access networking technologies, e.g., Long Range Wide Area Networks (LoRaWAN) [2], wireless local area networks (WLANs) based on the IEEE 802.11 standard (Wi-Fi) [3], wireless personal area networks (WPANs) based on the Bluetooth standard [4], etc. In the cellular domain, different communication generations could be used, e.g., ranging from 5G (i.e., the NarrowBand Internet of Things, NB-IoT, [5]) to 2G (i.e., the General Packet Radio

This publication has emanated from a research conducted with the financial support of the Bulgarian National Science Fund (BNSF) under the Grant No. КП-06-ИП-КИТАЙ/1 (КР-06-IP-СНINA/1) and the S\&T Major Project of the Science and Technology Ministry of China, Grant No. 2017YFE0135700.

I. Ganchev is with the University of Plovdiv "Paisii Hilendarski", Plovdiv, Bulgaria; the University of Limerick, Limerick, Ireland; and the Institute of Mathematics and Informatics, Bulgarian Academy of Sciences, Sofia, Bulgaria (corresponding author, e-mail: Ivan.Ganchev@ul.ie).

Z. Ji is with the North China University of Science and Technology, Caofeidian, Tangshan City, China (e-mail: Zhanlin.Ji@ieee.org).

M. O'Droma is with the University of Limerick, Limerick, Ireland (e-mail: Mairtin.ODroma@ul.ie).
Service, GPRS, [6]). Taking into account the great number of deployed IoT devices/nodes that need to be supported, the leading criterion here is the cost of the individual product, e.g., the so called Bill of Materials (BOM) needed to manufacture the end product. Based on our estimates, by the end of 2019, the 4G BOM was around 7 Euro, compared to 3 Euro for a $5 \mathrm{G}$ (NB-IoT) BOM. However, even though NB-IoT is fine for remote meter reading, its use in real-time communications seems problematic. Compared with these most recent cellular generations, $2 \mathrm{G}$ - with its BOM of about 1.5 Euro - is a very attractive wireless communication option for the IoT. With a carefully selected microcontroller unit (MCU), a 2G-based RTU can provide real-time communication while also operating in an ultra-low-power consuming mode. For instance, China Mobile Ltd. offers a very-low-price $2 \mathrm{G}$ eSIM card, with a $60 \mathrm{MB}$ annual data transfer for only 0.12 Euro. Perhaps this is one of the main reasons for increasing the number of the China Mobile's IoT connections by 290 million in 2019 [7]. So even now in the $5 \mathrm{G}$ era, $2 \mathrm{G}$ (GPRS) is still around and may continue to serve the IoT for several more years.

In [8], a GPRS-based DTU is proposed as a communication module for use in an agricultural irrigation and fertilization intelligent control system. Reference [9] proposes a water quality automatic monitoring system, utilizing a GPRS-based RTU. However, the power consumption of these and other similar units is at a mA level. In addition, as most of the utilized RTUs are commercial products, accessing and modifying their supporting software is not easy. Addressing this downside, in this paper, firstly a low-cost, ultra-low-power consuming and easily accessible RTU is proposed, based on the Cortex M3 microcontroller with a GPRS module. Then, taking into account the rapid development of the NB-IoT technology [10], a specially designed ultra-low-power consuming and low-cost NB-IoTbased RTU is proposed in the paper for use in IoT systems.

The aim is to use these RTUs in the elaborated generic multi-service cloud-based IoT operational platform, called EMULSION [11], and later develop and encompass other types of RTUs, allowing easy integration of different types of IoT (including constrained) devices with heterogeneous processing components and various communication capabilities and protocols. The goal is to allow 100,000 heterogeneous IoT devices to communicate simultaneously 
online with a single server. EMULSION will be also able to support communication and data exchange with various IoT applications.

The rest of the paper is structured as follows. Sections II and III present the GPRS-based RTU and NB-IoT-based RTU, respectively, designed for use in various IoT systems as described in Section IV. Conclusions are provided in Section V.

\section{GPRS-BASED RTU}

A GPRS-based RTU was developed for use, based on the MEDIATEK 6261 chipset, with a power management unit, and analog baseband and radio circuits. The interface of the complementary metal-oxide-semiconductor (CMOS) chipset includes a liquid crystal display (LCD), a keypad, a universal asynchronous receiver-transmitter (UART), SD, a universal subscriber identity module (USIM), a universal serial bus (USB) port, PWM, I2C, and regular I/O. Beside the 6261 chipset, the GPRS module includes a $26-\mathrm{MHz}$ crystal, a RF7198D IC, a ESD BDFN2C051VB, 5 electrical inductances, 29 chip capacitors, and 18 chip resistors. The PCB has 8 layers, as shown in Fig. 1a (the white line is the $50 \Omega$ impedance control line). Fig. $1 \mathrm{~b}$ depicts a joint board comprising up to 16 GPRS modules.

A Nucleus real-time operation system, developed by Accelerater Technology, Inc., runs on the MEDIATEK 6261 chipset.

\section{NB-IOT-BASED RTU}

The NB-IoT-based RTU consists of a direct current to direct current (DC-DC) power supply module, two low dropout (LDO) regulator modules, a NB-IoT module, an isolating TIA-485 module, an optical coupling isolation input module, and an Advanced RISC Machine (ARM) microcontroller unit (MCU). The MCU is a small system-ona-chip (SoC) computer. A variety of MCUs are produced, e.g., the Advanced RISC Machine (ARM) core processors, Atmel AVR, Texas Instrument TI MSP, Intel 8051, etc. Among these, the 32-bit ARM processors (licensed by the ARM Holdings PLC) are the most widely used. These include Cortex M0, M1, M3, M4, and M7. The ultra-low-power STM32L151 - with a 64KB Flash, 32MHz CPU - costs only $1 €$, but provides portable, scalable, reliable, and efficient solution for embedded software design. These are the reasons behind selecting it as the MCU for the designed NB-IoTbased RTU.

The hardware of the designed NB-IoT-based RTU includes three main modules:

1) MCU Module: This includes a 12-bit Analog-to-Digital Converter (ADC), and two ultra-low-power comparators. In a stop mode, it only consumes $1.2 \mu \mathrm{A}$. The 64-pin MCU version with a Low-profile Quad Flat Package (LQFP) was chosen for use;
2) NB-IoT Module: The Quectel BC28 NB-IoT was selected for this module due to its $1.77 \times 1.58 \mathrm{~cm}$ dimension, which meets the space requirement of the hardware design. Moreover, it only needs $500 \mathrm{~mA}$ current in a data burst mode and $3 \mu \mathrm{A}$ in a power safe mode with $3.3 \mathrm{~V}$ supply. The Universal Synchronous/Asynchronous Receiver/Transmitter (USART) serial port is utilized for communication with the MCU;

3) Power Supply Module: The power supply supports $7-14.4 \mathrm{~V}$ input. Two LDO provide $3.3 \mathrm{~V}$ VCC for the $\mathrm{MCU}$ and peripherals. A DC-DC unit provides $3.6 \mathrm{~V}$ VCC for the NB-IoT module.

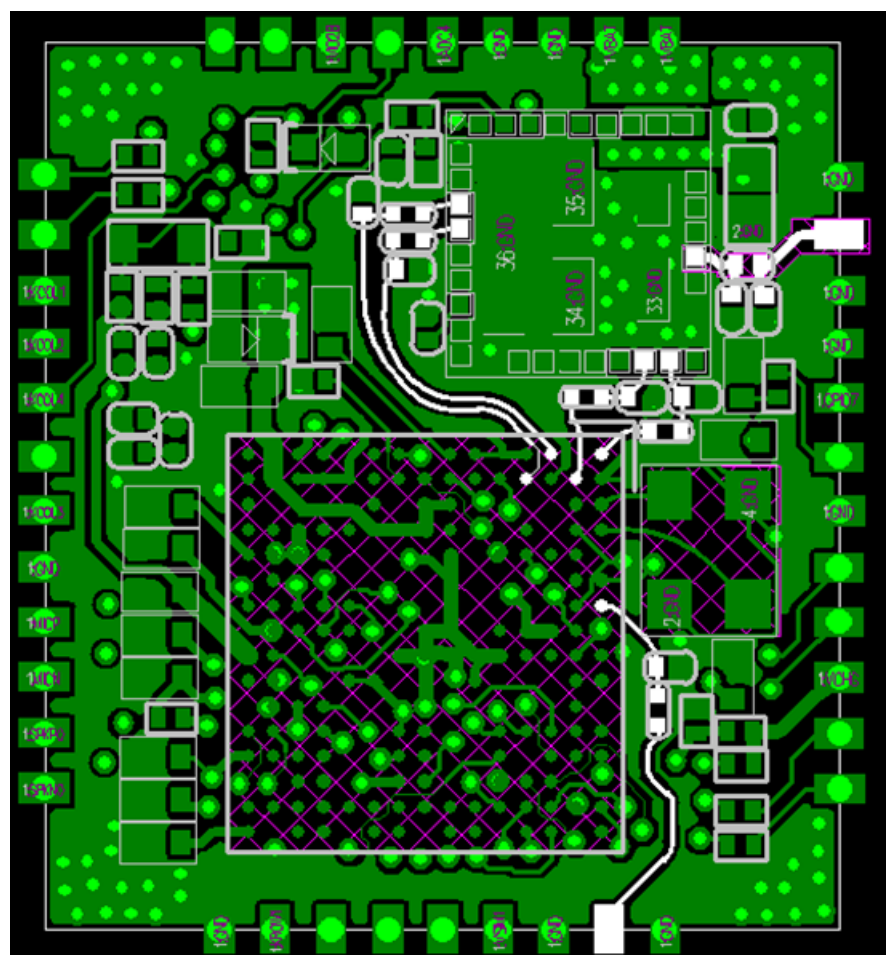

(a)

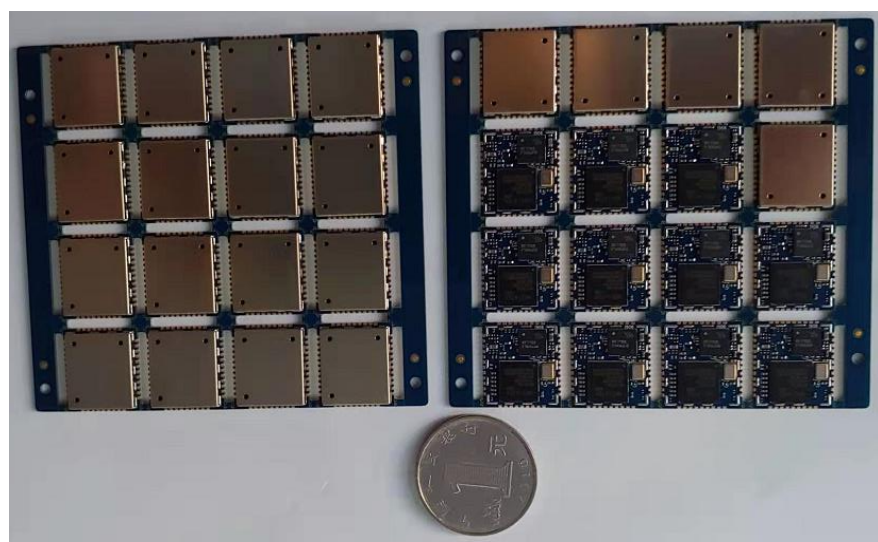

(b)

Fig. 1. (a) The PCB layout of the developed GPRS-based RTU; (b) 16 GPRS modules on a joint board. 


\section{A. MCU Module}

The STM32L151 schematic design is shown on Fig. 2. The MCU can be wakened up from a low-power sleep mode when receiving a rising edge on pin PA0 (I2C calendar) or pin PB5 (digital input). Then the MCU calculates the power voltage from pins PA1 and PA3. If the voltage can power up the sensors, the MCU enables the power MOSFET NTS4101PT1G via pin PB15 and collects data via the TIA485 serial port. After generating a data frame, the latter is sent to the NB-IoT module via the USART port. The MCU saves the data to EEPROM via the SPI-bus, i.e., pins PA5-PA7.

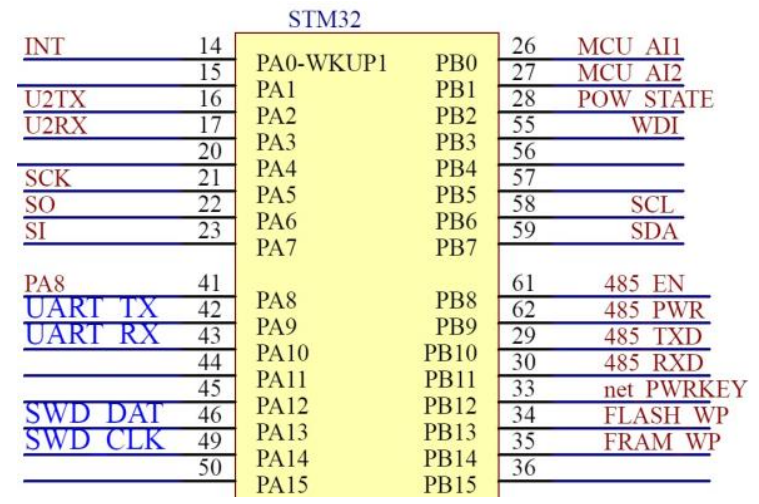

Fig. 2. The schematic design of the MCU for the NB-IoT-based RTU.

The I2C (pins PB6 \& PB7) connects the PCF8563 unit (which provides a CMOS real-time clock and calendar) with the MB85RC64 unit (which is a ferroelectric random access memory chip for saving the important parameters of the RTU), Fig. 3. The essential feature of PCF8563 and MB85RC64 is the low-power consumption as the operating supply current is at a $\mu \mathrm{A}$ level.
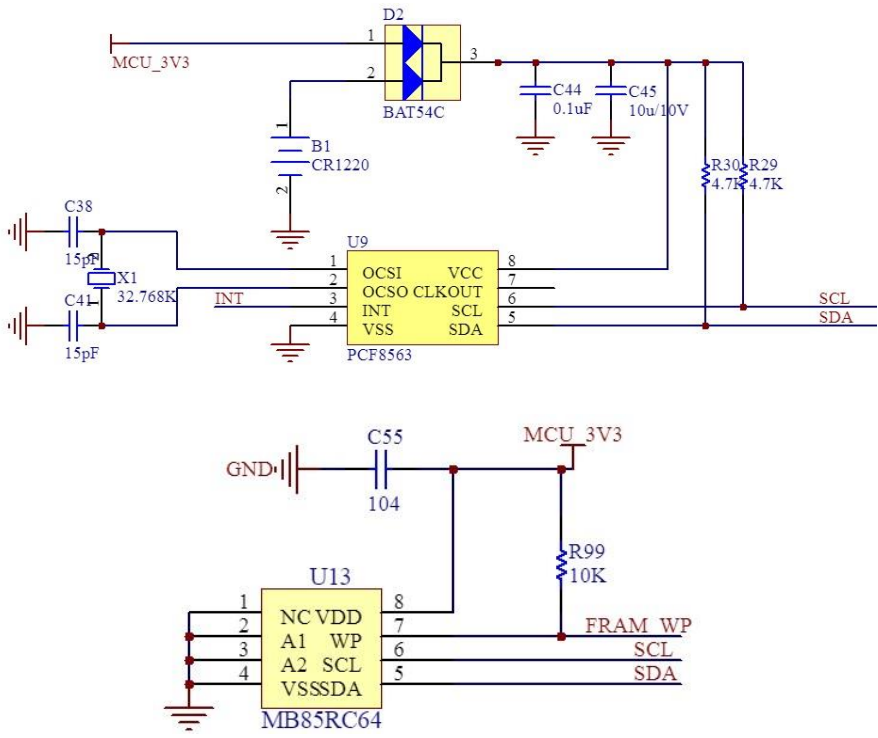

Fig. 3. The schematic design of the I2C for the NB-IoT-based RTU.
The TIA-485 serial port's schematic design is shown in Fig. 4. The power is controlled via pin PB9 by NTR4101.

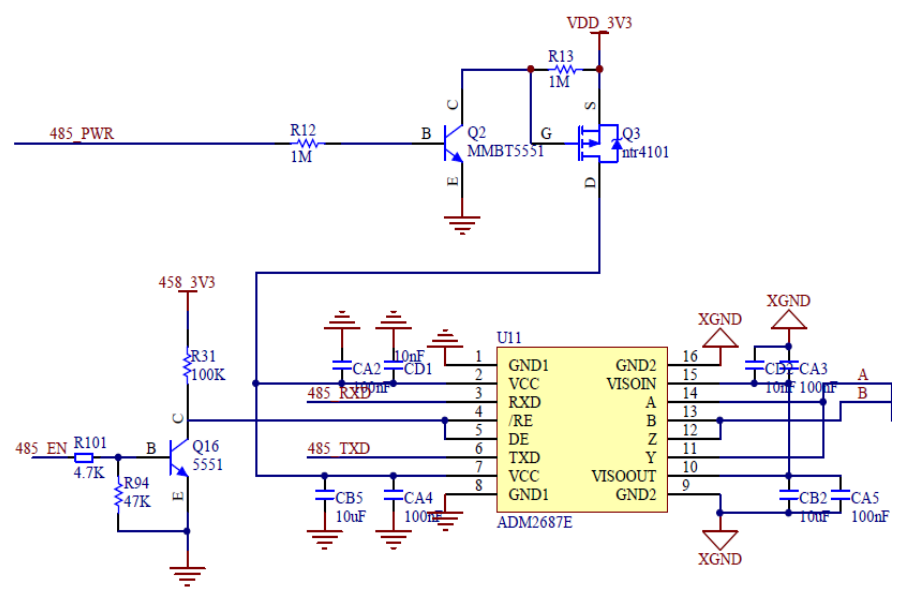

Fig. 4. The schematic design of the TIA-485 serial port for the NB-IoT-based RTU.

\section{B. NB-IoT Module}

The NB-IoT module is used for communication with the external world, facilitated by a $\mathrm{BC} 28$ unit that includes RX/TX ports for connection with PA2 and PA3, a GSM_ANT port for connection with the antenna, and a SIMports slot part for the SIM card. The power supply part includes four bypass capacitors - a $100-\mu \mathrm{F}$ tantalum capacitor and three ceramics capacitors (Fig. 5).

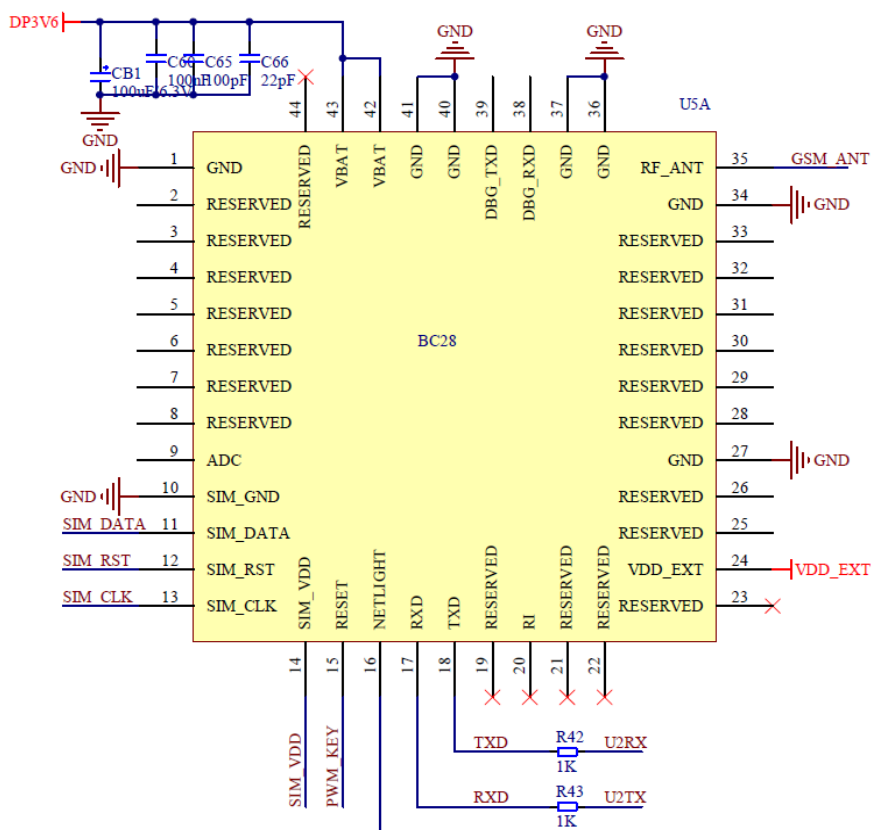

Fig. 5. The schematic design of the $\mathrm{BC} 28$ for the NB-IoT-based RTU.

\section{Power Supply Module}

Beside 3.3V LDOs, the NB-IoT module needs a 3.6V DC 
support. Fig. 6 shows the schematic design of the power supply module.

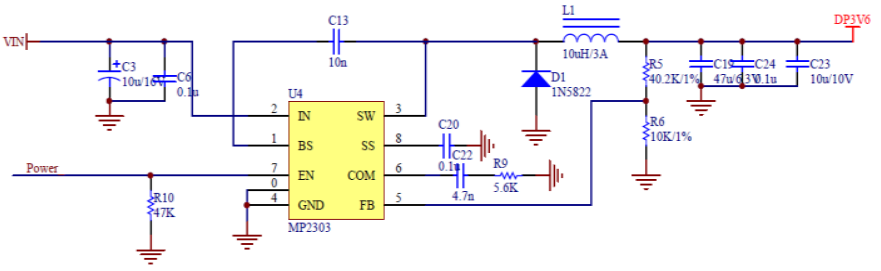

Fig. 6. The schematic design of the power supply module for the NB-IoT-based RTU.

The 4-layer NB-IoT RTU hardware PCB design is shown on Fig. 7. Its size is only $4 \times 10 \mathrm{~cm}$, which allows it to be easily inserted (along with the batteries) into small spaces. Four 3.6V dry batteries are used to achieve a 14.4-V (19-Ah) value.

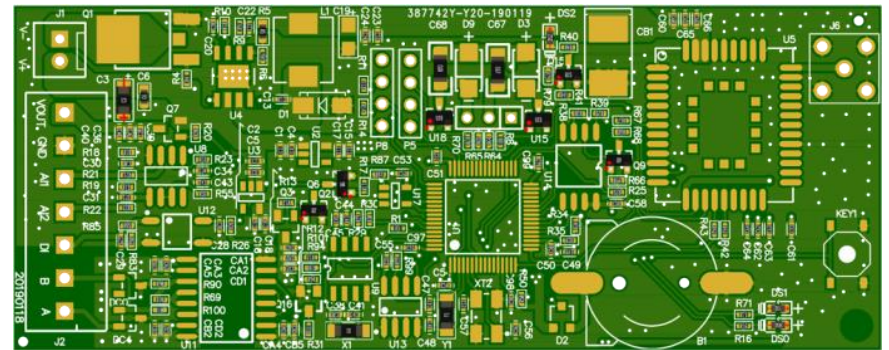

Fig. 7. The PCB design of the NB-IoT-based RTU.

The cost of the designed NB-IoT-based RTU is around 160 Chinese Yuans $(\sim 20 €)$. Fig. 8 shows the designed RTU running in an ultra-low-power consumption mode (the quiescent current is $7.5 \mu \mathrm{A}$ ).

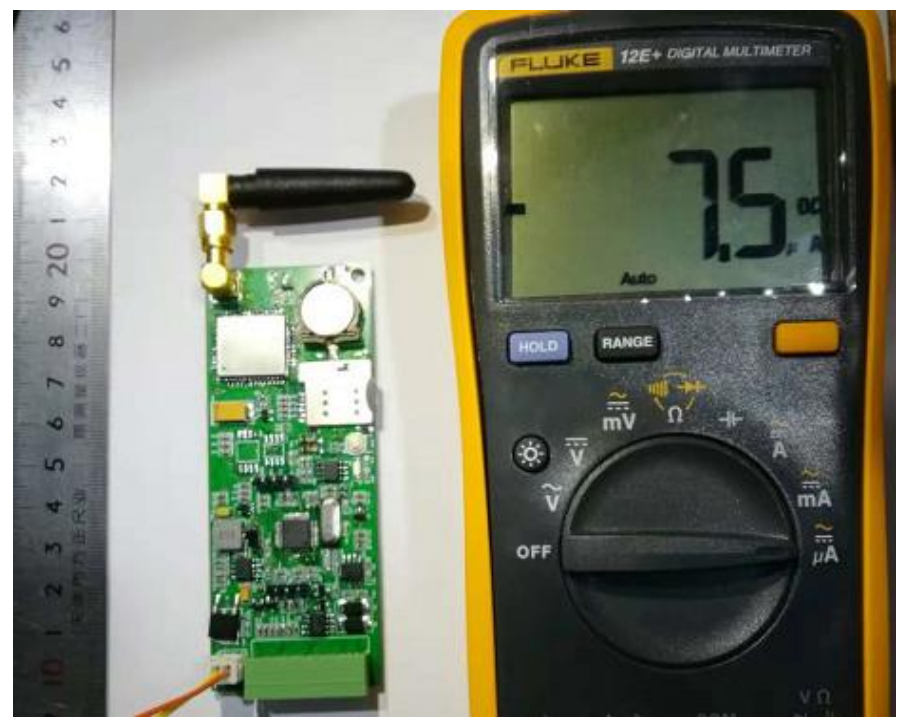

Fig. 8. The NB-IoT-based RTU running in an ultra-low-power consumption mode (with a quiescent current of $7.5 \mu \mathrm{A}$ ).

\section{Practical Use of Designed RTUs in Iot Systems}

The designed RTUs were successfully used in a number of IoT systems, including a heating control system, a smart electric boiler control system, a ground-water monitoring system, a pure water control system, a rented bicycles control system, etc. The utilized generic IoT system architecture is depicted in Fig. 9.

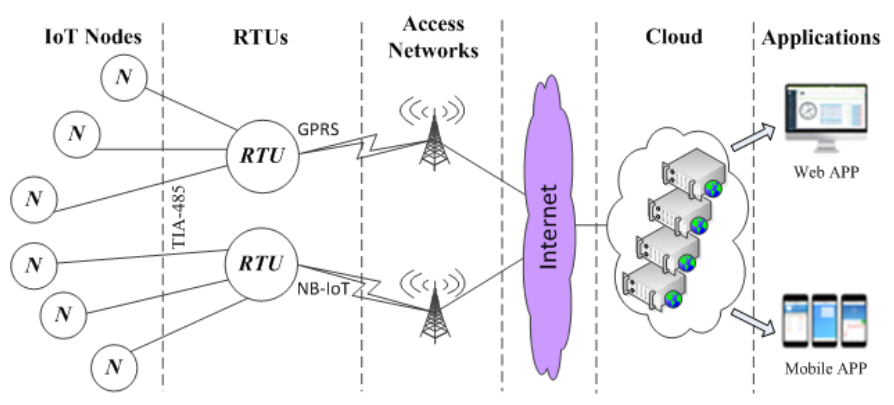

Fig. 9. The generic IoT system architecture used.

The IoT nodes (sensors, actuators, controllers, etc.) communicate with information centers (servers) in the cloud via the designed RTUs. Each RTU is equipped either with a GPRS or a NB-IoT module to reach the corresponding server(s) via wireless access networks and the Internet. To achieve robustness, each RTU can keep up to 8 TCP connections with different servers/ports. This way servers in the cloud collect and analyze data from all deployed RTUs in order to obtain respective commands/decisions/recommendations that need to be sent back to controllers, e.g., to regulate the temperature in the controlled areas or switch on/off electric boilers, etc.

In the RTU, the data is first encapsulated into a Modbus RTU message, shown in Fig. 10. The RTU adds a Modbus application (MBAP) header and sends the resulting application data unit to a server (in the cloud) via TCP. In the opposite direction, after receiving a message from the cloud, the RTU decapsulates the Modbus RTU message and processes the command, e.g., for RTU parameter updating, time synchronizing, water level gauge data reading, sensor parameters adjusting, etc.

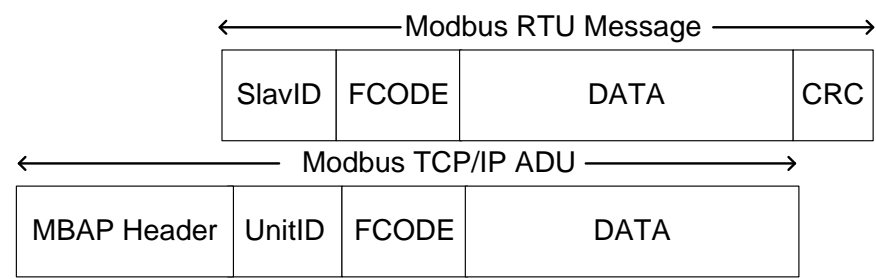

Fig. 10. The Modbus RTU message and the TCP/IP application data unit (ADU).

By December 2019 in China, there were 100,000 smart electric boiler control modules (Fig. 11a), 40,000 pure water controllers (Fig. 11b), and 50,000 rented bicycles, all 
equipped with the designed RTUs, and operating successfully as part of the aforementioned IoT systems.

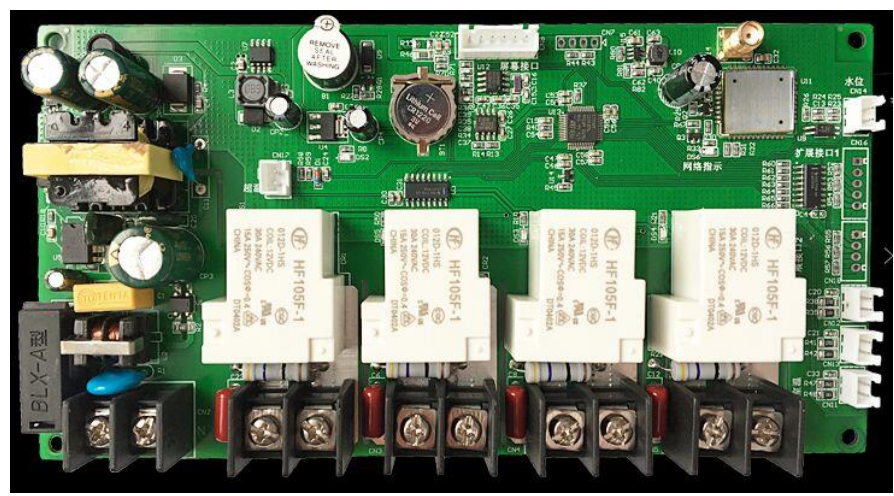

(a)

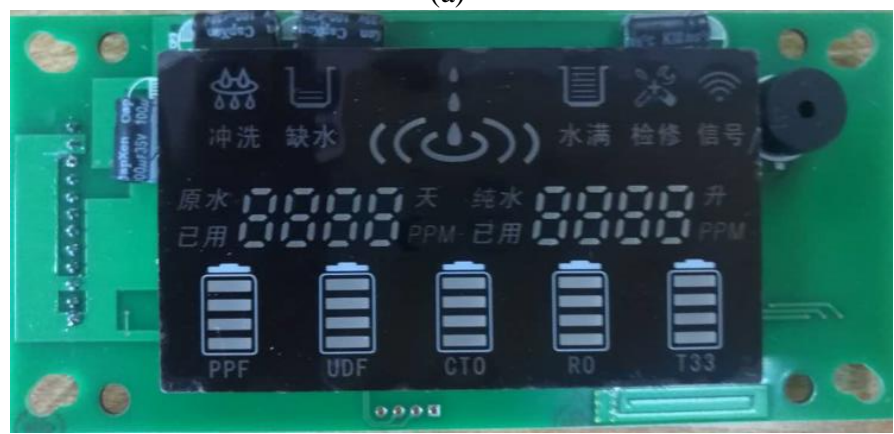

(b)

Fig. 11. (a) The developed smart electric boiler control module; (b) The developed pure water controller.

Fig. 12 shows a ground-water monitoring installation with a NB-IoT-based RTU installed in an Ingress Protection 68 (IP 68) box and hanged under the well lid. The RTU collects periodically (1 to 6 hours) a hydrologic data via a TIA-485 serial port and sends it to the cloud.

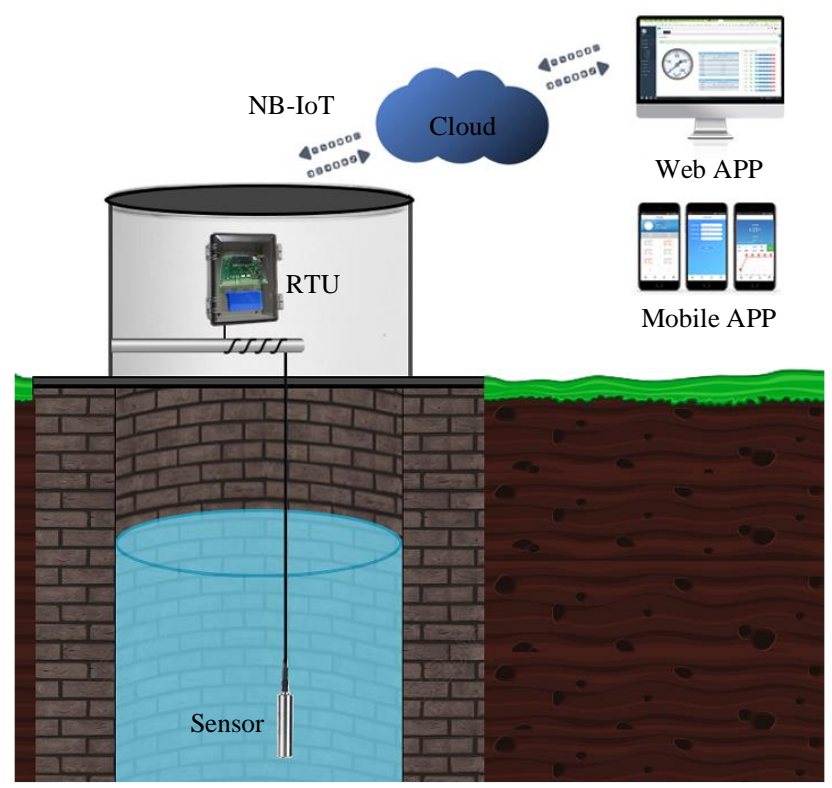

Fig. 12. A ground-water monitoring installation utilizing the designed NB-IoT-based RTU.
Fig. 13 depicts screenshots of the corresponding Android mobile application, developed for hydrologic monitoring.

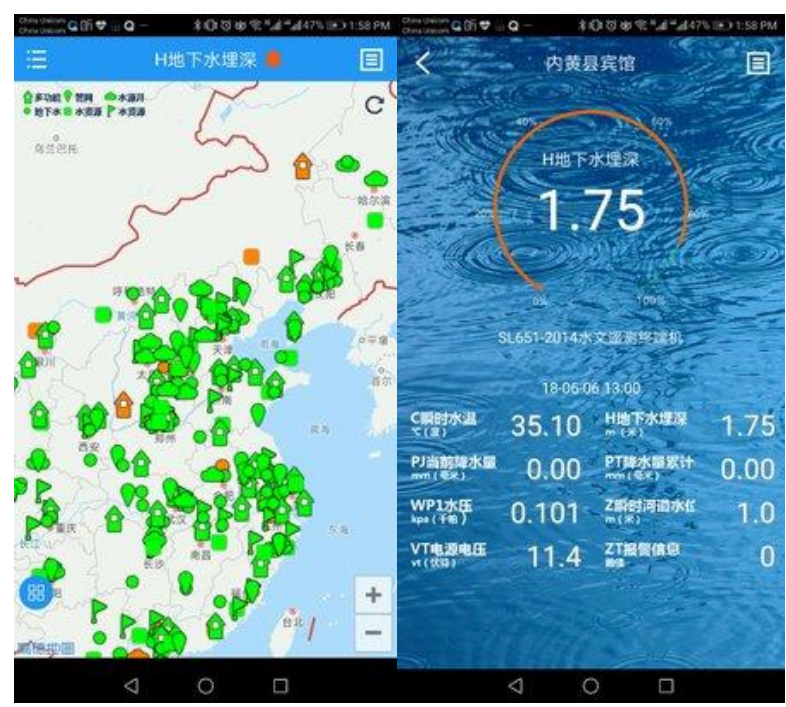

Fig. 13. Screenshots of the developed Android mobile application for hydrologic monitoring.

Fig. 14 depicts some results, obtained by the developed rented bicycles control system, showing the usage rate's dependency on time and weather conditions.
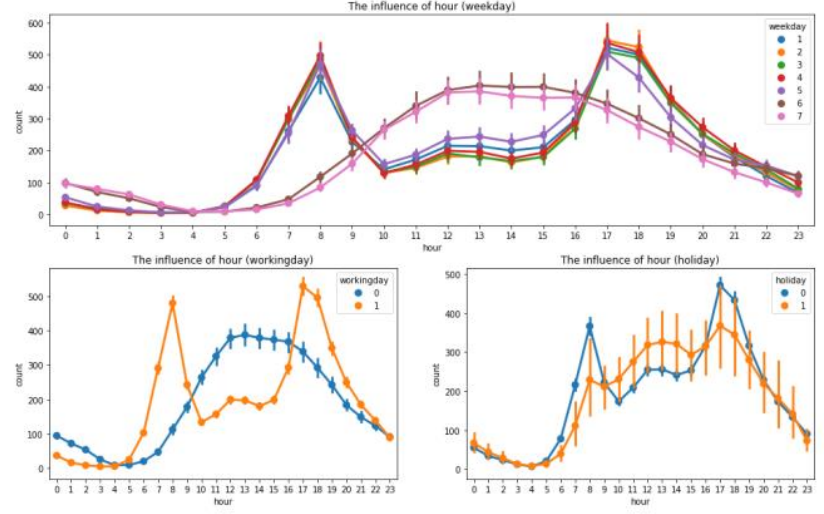

(a)
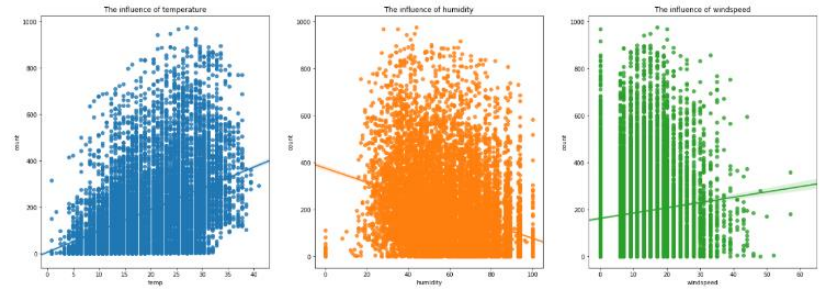

(b)

Fig. 14. The rented bicycles usage rate's dependency on: (a) Time; (b) Weather conditions.

Fig. 15 shows screenshots of the developed pure water control system, encompassing 40,000 pure water controllers connected to a single server and consuming only $70 \mathrm{MB}$ of its 
RAM and $2 \%$ of its CPU.

Fig. 16 shows screenshots of the developed Android mobile application for the smart electric boiler control system.

\section{CONCLUSION}

Designed low-cost and ultra-low-power consuming remote transfer units (RTUs) for use in a variety of IoT systems have been presented in this paper. The RTUs operate as communication gateways for collecting, aggregating, and sending IoT data to information centers (servers) in the cloud for further processing and data mining. Two types of RTUs, targeting different application scenarios and utilizing different communication standards, have been presented: (1) based on the General Packet Radio Service (GPRS) standard, and (2) based on the NarrowBand Internet of Things (NBIoT) standard. The developed RTUs were experimentally tested and their use was successfully demonstrated in a number of IoT systems in different areas, such as smart homes, smart transportation, smart environmental protection, etc.

\section{REFERENCES}

[1] WWAP, "The United Nations world water development report 4: Managing water under uncertainty and risk," ed: UNESCO Paris, 2012

[2] C. Zevenbergen, D. Fu, A. Pathirana, "Transitioning to Sponge Cities: Challenges and Opportunities to Address Urban Water Problems in China, Water, 10:1230, 2018.

[3] Y.-P. E. Wang, X. Lin, A. Adhikary, A. Grovlen, Y. Sui, Y. Blankenship, et al., "A primer on 3GPP narrowband Internet of Things," IEEE Comm. Mag., 55:117-123, 2017.

[4] Y. T. Wang, H. X. Zhang, J. C. Li, Y. Y. Wu, “Application and research of agricultural irrigation fertilization intelligent control system based on GPRS DTU," Applied Mechanics and Materials, 441:783-787, 2014.

[5] W. Dehua, L. Pan, L. Bo, and G. Zeng, "Water quality automatic monitoring system based on GPRS data communications," Procedia Eng, 28: 840-843, 2012

[6] China Mobile's 2019 Work Conference. https://iot.ofweek.com/201812/ART-132209-8120-30290220.html

[7] Y. Mehmood, F. Ahmad, I. Yaqoob, A. Adnane, M. Imran and S. Guizani, "Internet-of-Things-based smart cities: Recent advances and challenges, IEEE Comm. Mag., 55(9):16-24, 2017.

[8] I. Ganchev, Z. Ji, M. O'Droma, "Designing a cloud tier for the IoT platform EMULSION," WSEAS Trans. on Systems and Control, 14:375383. 2019.

[9] LoRa Alliance. "What is the LoRaWAN® Specification?," https://loraalliance.org/about-lorawan

[10] The Working Group for WLAN Standards. "IEEE 802.11 Wireless Local Area Networks. http://www.ieee802.org/11/

[11] Bluetooth Core Specification Working Group. "Bluetooth Core Specification," https://www.bluetooth.com/specifications/bluetooth-corespecification/

[12] GSMA. "Narrowband - Internet of Things (NB-IoT)," https://www.gsma.com/iot/narrow-band-internet-of-things-nb-iot/

[13] ETSI. " $2^{\text {nd }} \quad$ Generation:

GPRS, https://www.etsi.org/technologies/mobile/2g

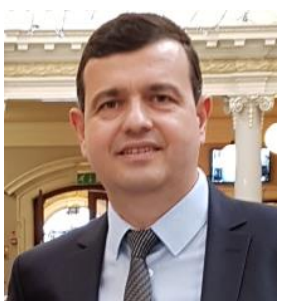

Ivan Ganchev (SMIEEE'08) is a Senior Member of the IEEE; the IEEE Communications Society; the IEEE Computer Society Technical Committees on: (i) Internet of Everything, (ii) Internet, (iii) Computer Communications, and (iv) Cloud Computing; the IEEE Communities on: (i) Future Networks, (ii) IEEE Internet of Things, and (iii) IEEE Smart Cities; and the IEEE Consultants Network. He received his doctoral and engineering (summa cum laude) degrees from the SaintPetersburg University of Telecommunications in 1995 and 1989, respectively. $\mathrm{He}$ is a Deputy Director of the Telecommunications Research Centre (TRC), University of Limerick (Ireland), an ITU-T Invited Expert, and an IET Invited Lecturer. He also holds a Full Professorship at the University of Plovdiv "Paisii Hilendarski".

Prof. Ganchev was involved in $40+$ international and national research projects. His research interests include: novel telecommunication and information paradigms; modelling, simulation, and emulation of complex telecommunication and information systems, networks, and services; informational modelling; future telecommunication networks and services; smart ubiquitous networking; Internet of Things (IoT); Internet of Services (IoS); Ambient Assisted Living (AAL); Enhanced Living Environments (ELE); Internet tomography; mHealth and mLearning ICT.

Prof. Ganchev has served on the Technical Program Committee of 300+ prestigious international conferences, symposia, and workshops. He has authored/co-authored 1 monographic book, 3 textbooks, 4 edited books, and $260+$ research papers in refereed international journals, books, and conference proceedings. He is an Area Editor of the Elsevier "Computer Networks" journal, an Associate Editor of the Wiley "Internet Technology Letters" journal, a Regional Editor (Europe) of the International Journal on Trust Management in Computing and Communications. He also seats on the Editorial Board of and has served as a Guest Editor for multiple international journals.

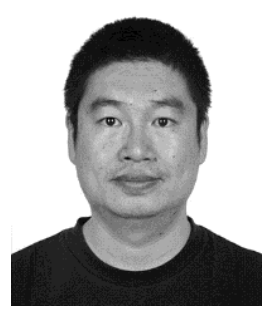

Zhanlin Ji received his MEng and $\mathrm{PhD}$ degree from Dublin City University and University of Limerick (Ireland) in 2006 and 2010, respectively. He is a Professor at the North China University of Science and Technology, China. His research interests include Internet of Things (IoT), cloud computing, big data management, and data mining. He has authored/coauthored over 60 research papers in refereed international journals, books, and conference proceedings.

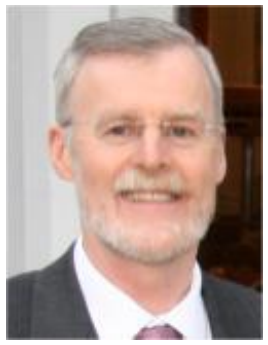

Máirtín S. O'Droma (SMIEEE'91) received his BE and $\mathrm{PhD}$ degrees from the National University of Ireland in 1973 and 1978, respectively. He is a Senior Academic and Honorary Director of the Telecommunications Research Centre (TRC), University of Limerick (Ireland). Dr O'Droma is an IEEE Subject Matter Expert, an ITU-T Invited Expert, a Fellow of the IET, and a President of the Irish Committee of Official Commission Members of the International Union of Radio Science (URSI). His research interests include: behavioral modelling, linearization \& efficiency techniques in multimode, multiband, multicarrier broadband nonlinear RF power amplifiers; complex wireless telecommunication systems simulation; wireless network and protocol infrastructural innovations for next generation wireless networks (wNGN), the ubiquitous consumer wireless world (UCWW) and cognitive networks. Dr O'Droma has authored/co-authored over 260 research publications in refereed journals, international conferences, and books. He served on the Technical Program Committee of numerous IEEE and other international conferences and workshops, and is on the editorial boards of several international journals. 

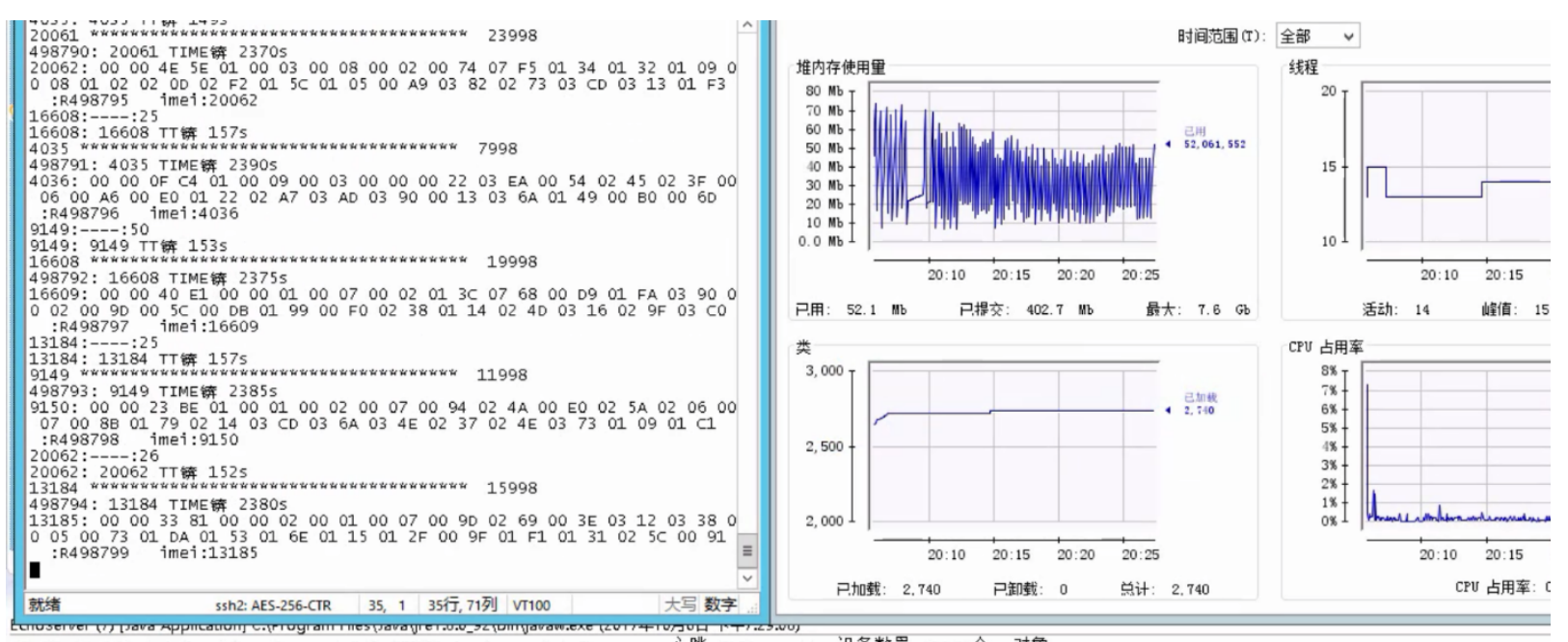

20:27:25.266 [n10EventLoopGroup-4-14] DEBUG 10.1 1anpu.EchoServer - 心跳: 9141 , nu11, 设备数墨: 38906 个, 对象

$20: 27: 25.206$ (n) 20:27:25.266 [nioEventLopgroup-4-7] DEBUG $779142:: \quad h b=9141,1,123,0,0,109,689,9,50,468,85,806,462,311,2017-10-06,20: 27: 25,7,0,0,0,683,257,385,113,755$.

20:27:25.266 [nioEventLoopGroup-4-14] DEBUG i0.1anpu. EchoServer - Channe1HandlerContext (EchoServer52005Handler2\#0, [id: 0xa620704b, L:/10.10.60.180:50055 . 20:27:25.266 [nioEventLoopGroup-4-14] DEBUG 10.1anpu. EchoServer - Channe1HandlerContext (EchoServer52005Handler \#0, [1d: 0xa620704b, L:/10.10.60.180:50055 -

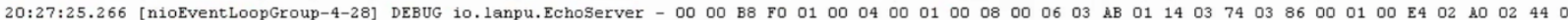
20:27:25.266 [nioEventLoopGroup-4-28] DEBUG 10.1anpu.EchoServer - 透传: Message: /60.2.39.228:5849:

Fig. 15. Screenshots of the developed pure water control system.

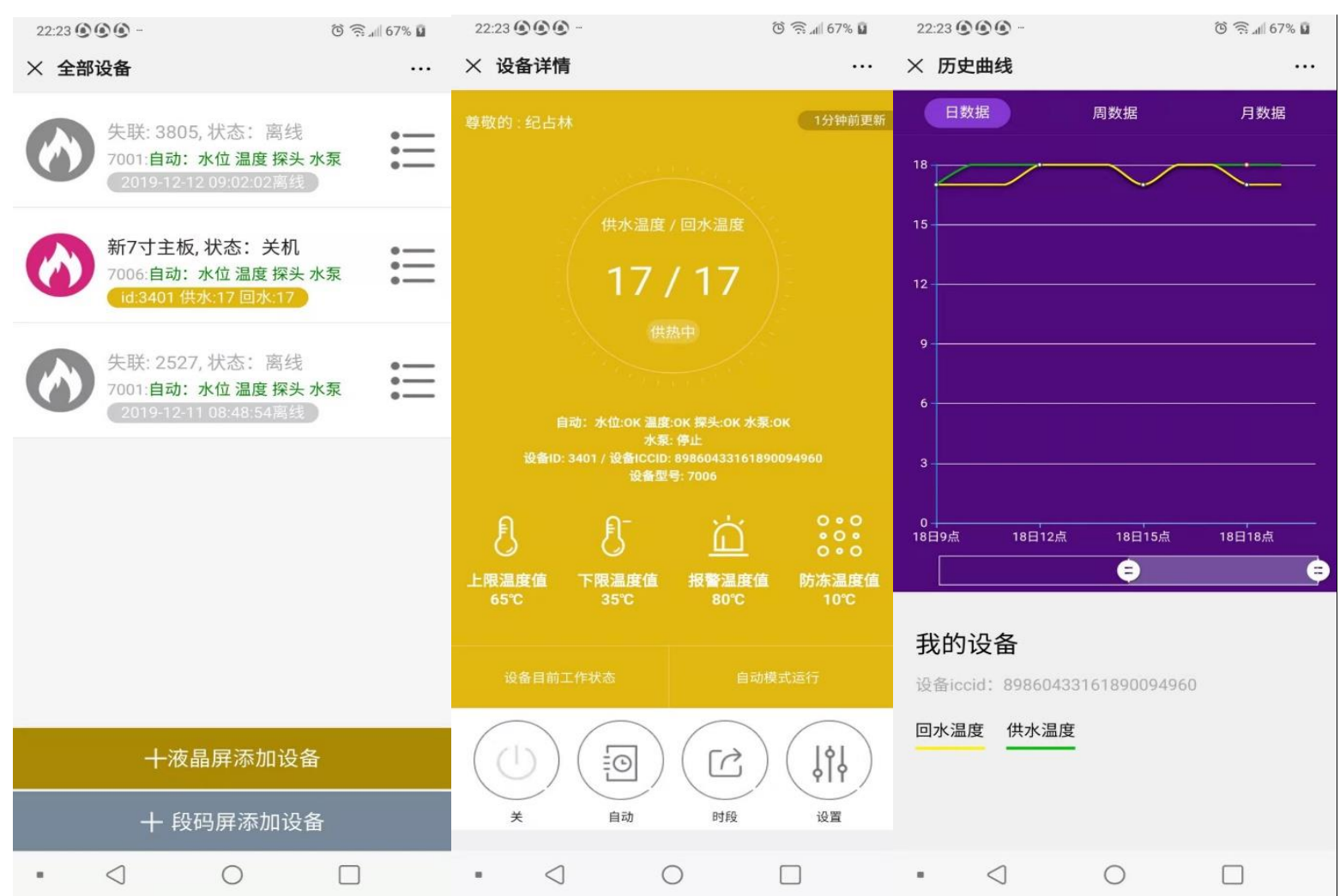

Fig. 16. Screenshots of the developed Android mobile application for the smart electric boiler control system. 